\title{
Psoríase e fatores de risco cardio- vascular: estudo observacional numa população urbana da Região Norte de Portugal
}

Inês Ferreira Santos Videira, ${ }^{1-2}$ Ana Raquel Marques, ${ }^{1,3}$ Ângela Cristina Pinto Neves, ${ }^{1,4}$ Débora Filipa Pimenta de Paiva Monteiro

\begin{abstract}
RESUMO
Objetivos: Vários estudos têm demonstrado a associação da psoríase com a diabetes mellitus (DM), hipertensão arterial (HTA), dislipidemia, tabagismo e obesidade. Neste trabalho pretende-se averiguar se existe associação entre psoríase e fatores de risco cardiovascular (FRCV) numa população urbana do norte de Portugal.

Tipo de estudo: Estudo observacional, transversal.

Local: Unidades Funcionais dos autores.

População: População urbana da Região Norte de Portugal.

Métodos: Foram incluídos todos os utentes com idade $\geq 18$ anos com o diagnóstico de psoríase em janeiro de 2015 das unidades funcionais dos autores. O grupo de comparação foi selecionado aleatoriamente numa proporção de 3:1. Colheram-se dados relativos ao diagnóstico de tabagismo, HTA, DM, obesidade e dislipidemia. Utilizou-se o teste qui-quadrado e o método de regressão logística para avaliar a existência de associação entre variáveis.

Resultados: Analisaram-se 1.980 utentes no total, sendo 51,4\% do sexo feminino e com média de idade de 49,7 anos (DP=17,2). Deste total, 495 utentes tinham o diagnóstico de psoríase. Da análise comparativa verifica-se no grupo com psoríase uma percentagem superior de tabagismo $(25,9 \%), \operatorname{HTA}(35,2 \%), \mathrm{DM}(15,6 \%)$, obesidade $(25,9 \%)$ e de dislipidemia $(28,9 \%)$ com significado estatístico, para um intervalo de confiança de $95 \%$. Após o controlo de fatores de confundimento verifica-se uma associação independente, estatisticamente significativa entre a psoríase e idade, género masculino, obesidade e tabagismo.

Conclusões: Este estudo mostrou uma frequência aumentada dos FRCV no grupo com psoríase. É importante enfatizar que apenas se estabeleceu uma associação e não uma relação de causalidade. No entanto, este trabalho alerta para a necessidade de identificação e controlo dos FRCV nos doentes com psoríase.
\end{abstract}

Palavras-chave: Psoríase; Obesidade; Hipertensão; Diabetes mellitus; Dislipidemia; Tabagismo

\section{INTRODUÇÃO}

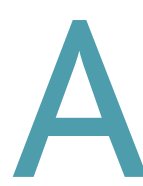

psoríase é uma doença inflamatória crónica que afeta predominantemente a pele, mas também as unhas e as articulações. ${ }^{1}$ Estimase uma prevalência entre 2 a $3 \% .^{2-3}$

Vários estudos verificaram a associação entre a psoríase e as comorbilidades que aumentam o risco cardiovascular, como sejam a diabetes mellitus (DM), ${ }^{4}$ hipertensão arterial (HTA), ${ }^{5}$ dislipidemia, ${ }^{6}{ }^{\text {tabagismo }}{ }^{7} \mathrm{e}$ obesidade. ${ }^{5}$ Outros estudos demonstram uma associa- ção entre a psoríase e o outcome cardiovascular, como a síndroma coronária aguda (SCA) e o acidente vascular cerebral (AVC) ${ }^{8} \mathrm{O}$ mecanismo exato ainda é incerto; contudo, os efeitos fisiológicos e psicopatológicos da

\footnotetext{
1. Médicas Especialistas de Medicina Geral e Familiar

2. USF Saúde em Família

3. UCSP São Mamede de Infesta

4. USF Lagoa

5. USF Pirâmides
} 
psoríase e os mecanismos patogénicos que envolvem a aterosclerose parecem ter um papel importante. ${ }^{9}$

A psoríase tem sido reconhecida como um distúrbio multissistémico, análogo a outras doenças inflamatórias imunes como a artrite reumatóide ou a doença de Crohn. Estas patologias partilham mecanismos patogénicos comuns, nomeadamente pela ativação de células dendríticas e linfócitos T, com libertação de citocinas, quimiocinas e fatores de crescimento. ${ }^{10} \mathrm{~A}$ psoríase está associada à elevação de marcadores sistémicos inflamatórios como a proteína $\mathrm{C}$ reativa. ${ }^{6}$ Por outro lado, já desde há muito tempo que a inflamação crónica tem sido implicada na etiopatogénese da aterosclerose, existindo múltiplos estudos que tentam explicar os seus mecanismos. ${ }^{11-13}$ Da mesma forma, as lesões ateroscleróticas apresentam infiltrados de linfócitos $\mathrm{T}$ que, quando ativados, induzem uma resposta inflamatória do tipo 1 (Th1). ${ }^{10}$

A maioria dos profissionais de saúde não reconhece a psoríase como associada a um risco cardiovascular desfavorável, apesar da evidência crescente para essa associação. ${ }^{6} \mathrm{O}$ risco aumentado de patologia cardiovascular observa-se em todo o espectro da gravidade da psoríase; contudo, parece ser mais importante nos doentes com psoríase grave, provavelmente decorrente de um estado inflamatório mais elevado. ${ }^{3}$ Os investigadores Mehta e colaboradore ${ }^{14}$ demonstraram que indivíduos com psoríase grave têm um risco acrescido de morte por evento cardiovascular, risco esse independente da existência de outros fatores de risco major tradicionais. Assim, a deteção precoce e a vigilância cuidada dos utentes com psoríase parece ser relevante para identificar e, eventualmente, prevenir outros fatores de risco cardiovascular (FRCV). Poderá, ainda, contribuir para a prevenção das complicações cardiovasculares ao instituir-se o tratamento para a psoríase mais precocemente, adequado à gravidade da doença, repercutindo, assim, na inflamação sistémica subjacente. ${ }^{6}$

Em Portugal ainda não existem estudos neste âmbito. Neste sentido, o objetivo deste trabalho é verificar se existe associação entre a psoríase e os FRCV considerados como tradicionais (obesidade, HTA, DM, dislipidemia e/ou tabagismo) numa amostra de utentes de quatro unidades funcionais (UF) dos cuidados de saúde primários (CSP) de uma região urbana no norte de Portugal.

\section{MÉTODOS}

Realizou-se um estudo observacional, transversal e analítico que teve início em março de 2014, com a elaboração do protocolo de investigação. Os autores tiveram aprovação dos conselhos clínicos da Unidade Local de Saúde Matosinhos (ULS) e do Agrupamento de Centros de Saúde (ACeS) Maia/Valongo, pela Unidade de Investigação Clínica e Comissão de Ética para a Saúde da ULS de Matosinhos e da Área Regional de Saúde do Norte e pelos coordenadores das UF.

O estudo decorreu na Unidade de Cuidados Personalizados (UCSP) São Mamede, Unidade de Saúde Familiar (USF) Lagoa, USF Pirâmides e USF Saúde em Família. Teve como população alvo os utentes inscritos nessas UF em janeiro de 2015, com médico de família, de idade igual ou superior a 18 anos ( $n$ total=53.535).

Relativamente à seleção dos participantes com diagnóstico de psoríase incluíram-se todos os utentes com idade igual ou superior a 18 anos, com diagnóstico de psoríase em janeiro de 2015 ( $n=495$ ), o que corresponde a uma prevalência de psoríase nas quatro unidades de estudo de $0,92 \%$.

A seleção do grupo de utentes sem psoríase foi realizada de forma aleatória através do programa random $\operatorname{org}{ }^{\circledR}$, a partir da população alvo, numa proporção de 3:1 $(n=1.485)$.

Definiram-se as seguintes variáveis: psoríase (codificação «Psoríase», S91, de acordo com a International Classification of Primary Care-2-ICPC-2), género ( $(\mathrm{Fe}-$ minino» ou «Masculino»), idade (em anos), obesidade (registo de IMC $\geq 30$, quantificado em quilogramas $/ \mathrm{m}^{2}$ ), HTA (codificação «Hipertensão com complicações», K87 e «Hipertensão sem complicações», K86, de acordo com a ICPC-2), DM (codificação «Diabetes Mellitus insulino-dependente», T89 e «Diabetes insulino-independente», T90, de acordo com a ICPC-2), Dislipidemia (codificação «Alteração do metabolismo dos lipídeos», T86, de acordo com a ICPC-2), Tabagismo (codificação «Abuso de Tabaco», P17, de acordo com a ICPC-2 e/ou registo de consumo de cigarros $\geq 1$ por dia e/ou qualquer outro registo de hábitos tabágicos).

As variáveis foram recolhidas com recurso ao Módulo de Informação e Monitorização das Unidades Funcionais (MIM@UF®) e aos registos clínicos do programaSClínico ${ }^{\circledR}$. A informação foi registada numa base de dados de uma folha de cálculo do programa Microsoft 


\section{QUADRO I. Quadro de abreviaturas}

\begin{tabular}{|c|c|c|}
\hline Abreviatura & Português & Inglês \\
\hline$\%$ & Percentagem & Percentage \\
\hline $\mathrm{ACeS}$ & Agrupamento de Centros de Saúde & Health Centers Grouping \\
\hline ARS & Administração Regional de Saúde & Regional Health Authority \\
\hline AVC & Acidente Vascular Cerebral & Cerebrovascular Accident \\
\hline CSP & Cuidados de Saúde Primários & Primary Health Care \\
\hline DM & Diabetes Mellitus & Diabetes Mellitus \\
\hline DP & Desvio-Padrão & Standard Deviation (SD) \\
\hline FRCV & Fatores de Risco Cardiovascular & Cardiovascular Risk Factors (CVRF) \\
\hline HDL & Do inglês, Lipoproteína de Alta Densidade & High Density Lipoprotein \\
\hline HTA & Hipertensão Arterial & Arterial Hypertension \\
\hline ICPC-2 & $\begin{array}{l}\text { Do inglês, Classificação Internacional dos Cuidados } \\
\text { Primários-2 }\end{array}$ & International Classification of Primary Care-2 \\
\hline IMC & Índice de Massa Corporal & Body Mass Index \\
\hline LDL & Do inglês, Lipoproteína de Baixa Densidade & Low Density Lipoprotein \\
\hline M & Média & Average \\
\hline $\mathrm{m}^{2}$ & Metro ao quadrado & Square meter \\
\hline MIM@UF & $\begin{array}{l}\text { Módulo de Informação e Monitorização das Unidades } \\
\text { Funcionais }\end{array}$ & $\begin{array}{l}\text { Information Module and Monitoring of the Functional } \\
\text { Units }\end{array}$ \\
\hline$n$ & Número & Number \\
\hline OR & Odds Ratio & Odds Ratio \\
\hline SCA & Síndrome Coronário Agudo & Acute Coronary Syndrome \\
\hline Sig & Significância & Significance \\
\hline Th1 & Resposta inflamatória do tipo 1 & Type 1 inflammatory response \\
\hline UCSP & Unidade de Cuidados de Saúde Personalizados & Custom Health Care Unit \\
\hline UF & Unidade Funcional & Functional Unit \\
\hline ULS & Unidade Local de Saúde & Local Health Unit \\
\hline USF & Unidade de Saúde Familiar & Unit Family Health \\
\hline Valor- $P$ & Nível descritivo & Descriptive level \\
\hline vs & Versus & Versus \\
\hline
\end{tabular}

Office Excel® , sendo cada participante identificado com um número interno de forma a garantir a confidencialidade dos dados. Na base de dados, cada coluna correspondeu a uma variável e cada linha a um indivíduo da população em estudo (com e sem psoríase).

Para a análise de dados recorreu-se ao programa Statistical Package for Social Sciences SPSS®, v. 22.0 para
Windows. A análise univariada foi utilizada para medidas descritivas (frequência absoluta, frequência relativa, média, desvio-padrão, mínimo, mediana e máximo) para caracterização da população. Para o estudo de proporções utilizou-se o teste qui-quadrado com o intuito de avaliar a existência de uma relação significativa entre as variáveis. Calcularam-se os Odds Ratio ajus- 
tados aos fatores de confundimento pelo método de regressão logística multivariável. O nível de significância estatística estabelecido foi de $5 \%$.

\section{RESULTADOS}

\section{Caracterização da amostra}

A amostra do presente estudo foi composta por 1.980 participantes, sendo esta maioritariamente do sexo feminino (51,4\%, $n=1.017)$, com idades compreendidas entre os 18 e os 97 anos $(\mathrm{M}=49,7 ; \mathrm{DP}=17,2)$. Da população analisada, 423 utentes $(21,4 \%)$ têm hábitos tabágicos, 527 (26,6\%) têm HTA e 219 (11,1\%) DM, $392(19,8 \%)$ obesidade e 498 (25,2\%) têm dislipidemia.

Do estudo comparativo entre os dois grupos de intervenientes (com psoríase $v s$ sem psoríase) constata-se uma diferença significativa em relação à idade dos participantes. Os participantes com psoríase apresentam uma idade média significativamente superior (53,0 anos; $\mathrm{DP}=14,7)$ relativamente à dos utentes sem esta patologia (48,5 anos; $\mathrm{DP}=17,8)$.

No que diz respeito ao género, a psoríase apresenta-se associada ao sexo masculino, resultado com significado estatístico, sendo a presença observada em $56,4 \%$ dos homens e em $43,6 \%$ das mulheres.

\section{Psoríase e FRCV}

Da análise comparativa verifica-se no grupo dos utentes com psoríase uma percentagem superior de tabagismo (25,9\%), HTA (35,2\%), DM (15,6\%), obesidade (25,9\%), dislipidemia (28,9\%), com significado estatístico para um intervalo de confiança de 95\% (Quadro II).

Ajustando a todas as variáveis, e no sentido de estabelecer uma associação independente, conclui-se que o género masculino, a idade, o tabagismo e a obesidade estão estatisticamente associados com a psoríase (Quadro III).

\section{DISCUSSÃO}

Neste estudo de base populacional observou-se uma frequência aumentada dos FRCV no grupo dos utentes com psoríase numa população urbana da região norte do país, verificando-se uma associação entre psoríase e género, idade, tabagismo, HTA, DM, obesidade e dislipidemia. Após a análise multivariada verificou-se que existe uma associação independente, estatisticamente significativa, entre psoríase e idade, género masculino, obesidade e tabagismo. No entanto, não se verificou uma associação independente com significado estatístico com HTA, DM e dislipidemia.

A prevalência aumentada de tabagismo nos doentes com psoríase neste estudo está de acordo com os resultados de outras investigações; contudo, a relação fisiopatológica entre o tabaco e a psoríase ainda não foi bem estabelecida. O impacto psicológico da psoríase, com aumento do estado de ansiedade ou mesmo condicionando a ocorrência de perturbações depressivas, poderá relacionar-se com o consumo de tabaco. Além disto, o tabaco poderá ser um fator de risco da psoríase,

\begin{tabular}{|c|c|c|c|}
\hline \multicolumn{4}{|c|}{ QUADRO III. Regressão logística para a psoríase } \\
\hline Variável & OR & IC & Valor-p \\
\hline Género masculino & 1,465 & $1,187-1,808$ & $<0,001$ \\
\hline Idade & --- & --- & 0,001 \\
\hline Tabagismo & 1,586 & $1,236-2,036$ & $<0,001$ \\
\hline HTA & 1,233 & $0,922-1,64$ & 0,157 \\
\hline DM & 1,167 & $0,834-1,634$ & 0,368 \\
\hline Obesidade & 1,390 & $1,070-1,806$ & 0,015 \\
\hline Dislipidemia & 0,903 & $0,698-1,168$ & 0,437 \\
\hline
\end{tabular}

Legenda: IC = intervalo de confiança; $D M=$ diabetes mellistus; $\mathrm{HTA}=$ hipertensão arterial; $\mathrm{OR}=$ odds ratio; Valor $-p=$ nível descritivo. 
com ação das substâncias tóxicas sobre a pele.,18

A associação encontrada com a obesidade está fortemente estabelecida em outros estudos. Alguns autores sugerem, como possível explicação, um estilo de vida pouco saudável. ${ }^{18}$ Por outro lado, a obesidade poderá estar envolvida na patogénese da psoríase, contribuindo para potenciar o estado inflamatório sistémico. ${ }^{3}$ Neiman e colaboradores, ${ }^{5}$ num estudo populacional de 2006, verificaram que o risco de obesidade nos doentes com psoríase era significativamente maior em comparação com o grupo de controlo e que se associava com a gravidade da doença.

A etiologia da psoríase ainda não está totalmente esclarecida, mas a evidência aponta para a interação de múltiplos fatores (genéticos, imunológicos e ambientais), ${ }^{10}$ sendo que a obesidade e o tabagismo, comorbilidades avaliadas neste estudo e com associação estatisticamente significativa com a psoríase, poderão contribuir para esta dermatose.

A associação entre a psoríase e a dislipidemia é controversa e com achados inconsistentes. No entanto, vários estudos, embora com limitações a considerar (e.g., amostras de pequenas dimensões e diagnósticos baseados em registos), demonstraram uma associação estatisticamente significativa entre a psoríase e um perfil dislipidémico com níveis elevados de colesterol total, triglicerídeos, colesterol LDL e níveis baixos de colesterol HDL. ${ }^{6,15-16}$ Um estudo de 2010, realizado em Israel, com uma amostra total de 36.787 indivíduos, dos quais 12.502 com diagnóstico de psoríase, verificou a existência de uma associação estatisticamente significativa entre a psoríase e HTA. ${ }^{17}$ Os resultados de outro estudo também evidenciaram uma associação estatisticamente significativa entre a psoríase e a DM. ${ }^{4}$ No entanto, o presente estudo não encontrou uma associação independente, estatisticamente significativa, entre a psoríase e dislipidemia, HTA e DM.

Algumas limitações devem ser consideradas. O presente estudo baseia-se em registos clínicos do SClínico® que poderão incluir uma codificação errada ou inexistente pelo médico assistente (viés de codificação). Por apenas ser considerada a codificação do diagnóstico de dislipidemia, HTA e DM nos registos clínicos pode acontecer não haver registo, mas existir um diagnóstico e de o utente estar medicado para as patologias em causa. A medicação em curso de cada uten- te selecionado não foi verificada pelos autores. Outra limitação do estudo foi o facto de os doentes com codificação de psoríase não terem sido examinados. Além disso, existem utentes com psoríase que mantêm seguimento hospitalar com pouco recurso aos CSP, não estando, por isso, codificado o diagnóstico. Finalmente, apesar de o nosso estudo sugerir que a dislipidemia, HTA e DM não apresentam associação independente com a psoríase, é possível que a não consideração de outros fatores de confundimento, ou mesmo a existência de fatores de confundimento desconhecidos atualmente, possam explicar a ausência de associação. Relativamente à DM, os estudos sugerem existir uma associação entre esta e a duração e gravidade da psoríase. Estes parâmetros não foram avaliados nos participantes incluídos no presente estudo. ${ }^{4}$

É importante enfatizar que este estudo apenas estabeleceu uma associação e não uma relação de causalidade. Estudos prospetivos futuros são necessários para estabelecer esta relação. Os autores sugerem que também será importante avaliar a associação com eventos cardiovasculares, como SCA e AVC.

No entanto, perante a evidência já existente, os médicos de família devem estar mais sensibilizados acerca do risco cardiovascular destes doentes e promover uma vigilância mais atenta. Os doentes com psoríase devem ser informados sobre a natureza complexa da sua patologia e incentivados a corrigir os FRCV modificáveis para uma redução do risco de doença cardiovascular e, em última instância, uma redução de um outcome cardiovascular desfavorável.

\section{AGRADECIMENTOS}

Os autores gostariam de agradecer a colaboração do Dr. Luís Alves, Dra. Joana Bastos, Dr. Pedro Couto, Dr. Pedro Mendes, Dra. Raquel Barros e Dra. Rosa Barreira pela orientação na metodologia do trabalho. Agradecem também à Dra. Liliana Pereira pela colaboração na análise estatística.

\section{REFERÊNCIAS BIBLIOGRÁFICAS}

1. Griffiths C, Camp R, Barker J. Psoriasis. In: Burns DA, Cox NH, Griffiths $C M$, editors. Rook's textbook of dermatology. 4th ed. Massachusetts: Blackwell Science; 2005. chapter 35.

2. Parisi R, Symmons DP, Griffiths CE, Ashcroft DM. Global epidemiology of psoriasis: a systematic review of incidence and prevalence. J Invest Dermatol. 2013;133(2):377-85.

3. Torres T, Sales R, Vasconcelos C, Selores M. Psoríase e doença cardiovascular [Psoriasis and cardiovascular disease]. Acta Med Port. 2013;26(5):601-7. Portuguese 
4. Cohen AD, Dreiher J, Shapiro Y, Vidavsky L, Vardy DA, Davidovici B, et al. Psoriasis and diabetes: a population-based cross-sectional study. J Eur Acad Dermatol Venereol. 2008;22(5):585-9.

5. Neimann AL, Shin DB, Wang X, Margolis DJ, Troxel AB, Gelfand JM. Prevalence of cardiovascular risk factors in patients with psoriasis. J Am Acad Dermatol. 2006;55(5):829-35.

6. Dreiher J, Weitzman D, Davidovici B, Shapiro J, Cohen AD. Psoriasis and dyslipidaemia: a population-based study. Acta Derm Venereol. 2008;88(6):561-5.

7. Mills CM, Srivastava ED, Harvey IM, Swift GL, Newcombe RG, Holt PJ, et al. Smoking habits in psoriasis: a case control study. $\mathrm{Br}$ J Dermatol. 1992;127(1):18-21.

8. Brauchli YB, Jick SS, Miret M, Meier CR. Psoriasis and risk of incident myocardial infarction, stroke or transient ischaemic attack: an inception cohort study with a nested case-control analysis. $\mathrm{Br} J$ Dermatol. 2009;160(5):1048-56.

9. Tablazon IL, Al-Dabagh A, Davis SA, Feldman SR. Risk of cardiovascular disorders in psoriasis patients: current and future. Am J Clin Dermatol. 2013;14(1):1-7.

10. Mrowietz U, Elder JT, Barker J. The importance of disease associations and concomitant therapy for the long-term management of psoriasis patients. Arch Dermatol Res. 2006;298(7):309-19.

11. Montero-Vega MT. The inflammatory process underlying atherosclerosis. Crit Rev Immunol. 2012;32(5):373-462.

12. Rosenfeld ME. Inflammation and atherosclerosis: direct versus indirect mechanisms. Curr Opin Pharmacol. 2013;13(2):154-60.

13. Ross R. Atherosclerosis is an inflammatory disease. Am Heart J. 1999;138(5 Pt 2):S419-20.
14. Mehta NN, Azfar RS, Shin DB, Neimann AL, Troxel AB, Gelfand JM. Patients with severe psoriasis are at increased risk of cardiovascular mortality: cohort study using the General Practice Research Database. Eur Heart J. 2010;31(8):1000-6.

15. Cohen AD, Gilutz H, Henkin Y, Zahger D, Shapiro J, Bonneh DY, et al. Psoriasis and the metabolic syndrome. Acta Derm Venereol. 2007;87(6):506-9.

16. Singh G, Aneja SP. Cardiovascular comorbiditiy in psoriasis. Indian J Dermatol. 2011;56(5):553-6.

17. Cohen AD, Weitzman D, Dreiher J. Psoriasis and hypertension: a casecontrol study. Acta Derm Venereol. 2010;90(1):23-6.

18. Doukaki S, Caputo V, Bongiorno MR. Psoriasis and cardiovascular risk: assessment by CUORE Project Risk Score in Italian patients. Dermatol Res Pract. 2013;2013:389031.

\section{FINANCIAMENTO}

Os autores assumiram o financiamento de todas as despesas decorrentes deste trabalho original.

\section{CONFLITO DE INTERESSES}

Os autores declaram não possuir quaisquer conflitos de interesse.

\section{ENDEREÇO PARA CORRESPONDÊNCIA}

Inês Ferreira Santos Videira

E-mail: ines_videira5@hotmail.com

Recebido em 20-12-2016

Aceite para publicação em 20-12-2017 


\section{ABSTRACT}

\section{PSORIASIS AND CARDIOVASCULAR RISK FACTORS: OBSERVATIONAL STUDY IN AN URBAN POPULATION OF THE NORTH REGION OF PORTUGAL}

Objectives: Several studies have demonstrated the association between psoriasis and diabetes mellitus, arterial hypertension, dyslipidemia, smoking and obesity. This paper aims to determine if there is an association among psoriasis and cardiovascular risk factors (CVRF) in an urban population of the north of Portugal.

Type of study: Observational, cross-sectional study.

Local: Healthcare units of the authors.

Population: Urban population of the North Region of Portugal.

Methods: There were included all patients from the healthcare units of the authors with $\geq 18$ years old and psoriasis diagnosis in January 2015. The comparison group was randomized at a ratio of 3:1. Data were collected relative to smoking, hypertension, diabetes mellitus, obesity and dyslipidemia. We used the chi-square test and logistic regression to assess the existence of association between variables.

Results: We analyzed 1,980 patients in total, $51.4 \%$ were female with the mean age of 49.7 years $(S D=17.2)$. Within this total, 495 patients had a diagnosis of psoriasis. From a comparative analysis, the group with psoriasis has a higher percentage of smoking (25.9\%), hypertension (35.2\%), DM (15.6\%), obesity (25.9\%), dyslipidemia (28.9\%) with statistical significance at the $95 \%$ confidence interval. After the control of confounding factors, we verified an independent statistically significant association concerning psoriasis and age, male gender, obesity and smoking.

Conclusions: This study showed an increased frequency of CVRF in the group with psoriasis. It is important to emphasize that it was only established an association, not a causal relationship. However, this study alerts to the need to identify and control the CVRF in patients with psoriasis.

Keywords: Psoriasis; Obesity; Hypertension; Diabetes mellitus; Dyslipidemia; Smoking 